\title{
IMPORTANCIA DE LAS HABILIDADES BLANDAS EN LA RETENCIÓN DEL TALENTO MILLENNIAL EN EL ÁREA DE VENTAS DEL SECTOR INMOBILIARIO EN LIMA METROPOLITANA
}

\author{
IMPORTANCE OF SOFT SKILLS IN THE RETENTION OF \\ MILLENNIAL TALENT IN THE SALES AREA OF THE REAL ESTATE \\ SECTOR IN LIMA METROPOLITAN
}

\author{
María Adela Cruz Aguilar ${ }^{1}$ Luz María Díaz Herco²
}

\begin{abstract}
Resumen
Este artículo estudia los determinantes de la retención del talento millennial del área de ventas en el sector inmobiliario peruano. Para lo cual, se aplica un diseño metodológico cualitativo por medio del estudio de caso, estudiando a una empresa peruana del sector inmobiliario. En el estudio se aplicó entrevistas, basadas en las necesidades de información resultado del estudio exploratorio y de la revisión de literatura, a colaboradores de distintas áreas para lograr la saturación y triangulación de resultados. Como principales hallazgos del estudio, se encontró que los colaboradores de la generación millennial valoran el ambiente favorable de trabajo y oportunidades de crecimiento dentro de la organización como criterios de retención laboral.
\end{abstract}

Códigos JEL: M12, M54

\begin{abstract}
This article studies the determinants of the retention of millennial talent from the sales area in the Peruvian real estate sector. For which, a qualitative methodological design is applied through the case study, studying a Peruvian company in the real estate sector. In the study, interviews were applied, based on the information needs resulting from the exploratory study and the literature review, collaborators from different areas to achieve saturation and triangulation of results. As the main findings of the study, it was found that the collaborators of the millennial generation value the favorable work environment and opportunities for growth within the organization as criteria for job retention.
\end{abstract}

\footnotetext{
${ }^{1}$ Programa de Administración de Empresas - Universidad de Piura, (Perú).

E-mail: maria.cruz.2196@gmail.com

2 Programa de Administración de Empresas - Universidad de Piura, (Perú).

E-mail: luzmadiaz955@gmail.com

Nota: Las autoras agradecen a la profesora Valentina Gomes Haensel Schmitt por la asesoría en la investigación y revisión del texto para su publicación.
} 


\section{INTRODUCCIÓN}

A lo largo de la última década, se ha estudiado con mayor detenimiento el comportamiento de los millennials en el ambiente laboral a nivel mundial. Esto se puede atribuir al interés que genera el actuar y pensar que tiene esta generación, ya que surgen cambios en el ámbito laboral conforme mayor cantidad de millennials se unan a la fuerza laboral. Estudios han proyectado que para el 2020 y el 2030, los miembros de esta generación representaran 50\% y $35 \%$ respectivamente de la fuerza laboral $(\mathrm{PwC}$, 2014; CEPLAN, 2015). De esta forma, se puede decir que esta será la siguiente generación en liderar la fuerza laboral, y la que haciendo uso de su nueva mentalidad y características lograrán potenciar el éxito de las empresas en la que se desarrollen (Myers \& Sadaghiani, 2010; Huyler, Pierre \& Ding, 2015).

En países latinoamericanos como el nuestro, los estudios han sido alrededor de los rasgos diferenciadores entre los pertenecientes a esta generación y las previas como la $\mathrm{X}$ o los babyboomers. Estos concluyeron que a los millennials les es más valioso contar con un ámbito laboral que les permita interactuar con libertad entre colaboradores, promueva la libertad en la toma de decisiones, colaboración y continuo feedback 360; siempre buscando el sentido del trabajo que realizan (Paulone \& Pulice, 2012; Goyenechea, 2016; Acosta, 2017; Lima \& Polanco, 2017).

Así mismo se encuentran estudios enfocados en las diferencias dentro de este cohorte generacional, de forma que se pueden diferenciar sub-grupos definidos por las facetas en las que el sujeto se encuentre. De esta manera se ha podido observar dos sub-grupos: quienes carecen de independencia económica, sin claro objetivo de vida ni búsqueda de compromiso en sus relaciones personales; o quienes la han conseguido, poseen metas claras y un sentimiento de compromiso tanto en su vida personal como laboral (Gordon, 2016). Son estos últimos los que son miembros activos del mercado laboral, abriendo un nuevo campo de estudio.
En Perú, las investigaciones respecto a la faceta laboral de los millennials se centran mayoritariamente en sectores como el financiero (Bergerman \& Tantaleán, 2016; Meza, 2016) y el sector retail o de ventas (Pacheco \& Ríos, 2017; Salas, 2017). Uno de los factores del extenso estudio en estos sectores es la alta rotación según La Asociación Peruana de Recursos Humanos; sin embargo, sectores como minería, energía, petróleo e inmobiliaria son los que presentan mayor dificultad en la atracción y retención del talento según la consultora de reclutamiento Cornerstone Lima (La República, 2016).

En el estudio "Tendencias de retención del talento en Perú" se señala que sólo el $50 \%$ de las empresas consiguen el nivel de rotación por debajo del nivel deseado de menos de $10 \%$ o $5 \%$ (PriceWaterhouseCoopers, 2014). Por lo que el ambiente corporativo actual deberá modificar su comportamiento y rediseñar sus estructuras para subsistir en el futuro (Pierce, 2018). Este cambio implica que la combinación de habilidades y características buscadas en el perfil del colaborador sean adaptadas a las nuevas demandas del mercado laboral.

La generación millennial ha contado a lo largo de su crecimiento con el Internet, una de las mejores herramientas en la búsqueda del conocimiento. Por tal motivo, las habilidades blandas como la capacidad de relacionarse con los demás, van tomando mayor importancia sobre las habilidades duras, las cuales se refieren a todos los conocimientos que pueden ser adquiridos a través del estudio (Stasz, 2001). En este estudio, se tratarán las siguientes habilidades como principales en el desarrollo del millennial en el ambiente laboral: orientación al conocimiento, liderazgo, valores éticos, capacidad para trabajar en equipo $y$ finalmente las habilidades comunicativas.

Esta investigación se enfoca en la retención del talento millennial del área de ventas en el sector inmobiliario peruano. Este sector tiene una rotación lenta de ventas, el promedio de tiempo que demora efectuar una es de 19.3 meses y es en gran medida influido por las políticas del país (ADI Perú, 2018). La empresa peruana de estudio 
tiene más de 10 años en el mercado, enfocada en la construcción y venta de edificios residenciales.

Son tanto los factores de cambio de mentalidad de los millennials como las dificultades que presenta este sector, cuestionando si al darles mayor importancia a las habilidades blandas al momento de la selección y retención de personal se tendría un efecto positivo en la retención de talento millennial. Y para conseguir este objetivo se identificaron las habilidades clave en la retención, los elementos buscados por la generación Y, el grado de influencia de las habilidades blandas en los trabajadores millennial tanto en el ambiente laboral como en el desarrollo profesional y los factores clave en la retención efectiva de estos trabajadores.

Se debe tener en cuenta que los resultados obtenidos no pueden ser generalizados, debido a ser este un estudio de caso. Sin embargo, el tema puede ser replicado en otras empresas que presenten características similares, considerando que los resultados podrían ser afectados por los elementos del entorno que sucedan durante dichas investigaciones.

\section{HABILIDADES BLANDAS Y RETENCIÓN DEL TALENTO HUMANO}

La importancia de las habilidades blandas en el ambiente laboral viene siendo parte fundamental en el desarrollo de las personas para el crecimiento de las empresas. El 85\% del éxito en el trabajo se debe al desarrollo de habilidades blandas de los trabajadores y el $15 \%$ restante a las habilidades técnicas (National Soft Skills Association, 2015).

Las habilidades blandas son un grupo de habilidades no cognitivas, pueden ser llamadas habilidades del "saber ser" debido a que son necesarias para realizar actividades tan propias de la persona como el relacionarse con otros. Al momento de trabajar con otras personas se necesita de habilidades interpersonales, personales, de grupo y de organización para poder realizar las tareas del día a día con éxito (Barreto \& Izquierdo, 2000; Levasseur, 2013).
El desarrollo de estas habilidades blandas es de manera continua, a través de la práctica y de la retroalimentación, logrando que la persona se desenvuelva y mejore en cada actividad. Son necesarias en personas que tienen a cargo un equipo o suelen desarrollar su trabajo en equipos, habilidades como liderazgo, comunicación ya sea verbal o no verbal, de organización y habilidades cognitivas, entendidas como resolución de problemas, manejo de situaciones, entre otras; sin olvidar que este desarrollo inicia porque hay una motivación personal por aprender y el ambiente es favorable para el aprendizaje (Levasseur, 2013; Weber et al., 2009). Pues el éxito de los millennials en el ambiente laboral depende de la educación y del aprendizaje que le brinde la organización (Hershatter \& Epstein, 2010).

Hoy, en el mundo laboral lleno de competitividad no basta con tener habilidades duras, ya que solo estas no garantizan el éxito (Maya, 2016). Y las habilidades en general van cambiando con el tiempo, por ejemplo, la habilidad de un supervisor cada vez está más enfocada en la capacidad de innovación ya sea de procesos, la forma de trabajo o en la mejora del servicio para el cliente; también, busca un verdadero equipo de trabajo y que sea capaz de influir en ellos para lograr un mejor resultado (Pearlman \& Barrett, 2013).

Puga \& Martínez (2008) realizan una investigación de todas las teorías sobre las competencias que deberían tener los directivos. En este estudio muestran las habilidades que debería tener todo directivo en el 2008, pero en la actualidad, estas habilidades blandas son necesarias no solo en los directivos sino en todo el personal, para que la empresa tenga un crecimiento consistente. De acuerdo con los autores las principales habilidades blandas son: orientación al conocimiento, liderazgo, habilidad para comunicar, valores éticos y capacidad para trabajar en equipo. Es decir:

1. Orientación al conocimiento, esta habilidad se refiere a la capacidad de aprender, poder actuar en distintas situaciones y adaptarse al cambio. Estos aprendizajes se deben dar de forma individual y grupal para que sean 
efectivos y completos. Requiere de una mejora continua y un clima laboral que permita y facilite el aprendizaje (Ahmed, Loh \& Zairi, 1999).

2. Liderazgo, son todas las actividades que motivan en dirección al desarrollo del propósito que tiene la empresa. El líder tiene que ser capaz de lograr que las personas a su cargo actúen por el bien de toda la empresa. Se requiere que el líder sea responsable, justo, equitativo, que pueda ser autocrítico, veraz y quiera el desarrollo de todas las personas, es decir, de la empresa en su totalidad (Pérez López, 1997).

3. Habilidad para comunicar, es la capacidad que tiene una persona para transmitir de manera asertiva y directa el mensaje que desea. Es necesario no solo saber transmitir, sino también escuchar. Muchos afirman que la habilidad de comunicar es la más importante en el ambiente laboral, Stasz (2001) le da énfasis que es distinto para cada puesto de trabajo; sin embargo, lo que siempre se debe mantener es que la persona logre expresar lo que desea, resumiéndolo en tres palabras: dar, recibir y entender el mensaje.

4. Valores éticos, al entender la empresa como una organización de personas, un lugar donde van a desarrollarse y lograr una buena sociedad genera un valor trascendente, logrando sostenibilidad en el tiempo.

5. Capacidad para trabajar en equipo, para lograr los objetivos globales es necesario el compromiso, ya que de ello depende el desempeño de la empresa y la calidad del servicio ofrecido. El objetivo es mejorar los resultados mediante el aporte de cada miembro que esté dispuesto a dar lo mejor en base al bien común.

Asimismo, la retención del talento humano es el conjunto de políticas que la empresa pone en acción con el objetivo de mantener a sus empleados dentro de la compañía y así disminuir el índice de nivel de rotación de personal.
El mayor reto del área de recursos humanos ahora no solo es encontrar el talento humano, sino que este se quede en la empresa, logrando identificarse con ella. El área debe cambiar continuamente y mantenerse a la par con los cambios del mundo (Chiavenato, 2009; Latukha, 2011). Y con esto se debe entender que la empresa también deberá cambiar. No es traído solamente por el uso de nuevas tecnologías o modelos, sino por la entrada de nuevas actitudes y mentalidades, en otras palabras, la entrada de nuevo talento, y retenerlo cuando ya se tenga.

Las personas tienden a salir de un trabajo porque no están satisfechos con sus responsabilidades y a veces porque el ambiente laboral tampoco es favorable. Usualmente las personas entre 18 y 29 años son las que cambian con facilidad de empresas ya que están la búsqueda de un trabajo en el que puedan aportar y viceversa, observando no solo beneficios económicos, sino desarrollo profesional, comodidad en el ambiente de trabajo y la cultura organizacional (Allen et al., 2010). Los factores para una efectiva retención del talento humano son ambiente laboral y el desarrollo profesional (Guerrero et al., 2006; Férnandez, 2002). Esto es:

\section{Ambiente Laboral}

Los trabajadores van a realizar su labor, donde se relacionan con diferentes elementos externos que intervienen en sus actividades cotidianas. Indican que el impacto que tienen estos elementos en el trabajador, de ser negativo, podrían llegar a afectar la salud - mencionan Guerrero, Cañedo, Rubio, Cutiño \& Fernández (2006). Así mismo señalan que para que el trabajador se desarrolle adecuadamente y pueda tener resultados de calidad, los elementos del ambiente laboral deberán buscar el bienestar mental y físico del colaborador. Esto nos denota, la necesidad de un buen ambiente laboral para el aumento de la eficiencia de los trabajadores, así como su bienestar personal y de la compañía. De tornarse tóxico este ambiente, lo más probable es que el trabajador busque salir pronto. 


\section{Desarrollo profesional}

La forma más frecuente de desarrollarse profesionalmente es obtener un ascenso. Sin embargo, Fernández Llosa, N. (2002) indica que la rotación de puestos de forma interna y los movimientos laterales de puesto también son formas de desarrollo profesional, ya que cada puesto requiere de diferentes habilidades. También, se establece una relación directa entre el desarrollo profesional y el aprendizaje que se va adquiriendo.

La retención del talento en el ambiente laboral, hoy en día, no solo depende de los incentivos económicos sino de otros que ayuden a cada colaborador a ser mejor. Hay diferencias en cada cohorte generacional, para llevar este cambio con éxito es necesario desarrollar una estrategia, teniendo en cuenta las habilidades y capacidades de los trabajadores junto con el objetivo que se quiere lograr (Zopiatis et al., 2012).

En especial, para Latukha (2011) el talento millennial es el más complicado de retener que las generaciones anteriores, como mencionamos anteriormente, por el estilo de vida y el entorno versátil, pero que es necesario para lograr mejores resultados y creación de valor de la empresa, porque al final las empresas son desarrolladas por y para personas. Entrevistó a personas recién graduadas de 30 empresas que habían dejado de trabajar y los factores fueron porque no sentían que tenían retos, no había posibilidad de desarrollo a futuro, no encontraron relación con el ambiente laboral y no había un balance entre el trabajo y la vida personal.

\section{MILLENNIAL EN EL AMBIENTE LABORAL}

El millennial es aquella persona nacida entre el 1980 y 1995. Siendo así parte de esta investigación, colaboradores entre los 23 y 38 años (Finn, D. \& Donovan A., 2013). También, son llamados millennials, las personas a quienes “el Internet ha 'atravesado' su vida desde la niñez o la adolescencia", junto con la visión de carpe diem y la disposición a movilizarse laboralmente, son tres de las características más resaltantes.
El millennial prefiere trabajar cómodo desde su hogar, de acuerdo a su horario, y consideran que el desarrollo profesional va junto con la pasión. Son personas que buscan crecer en el trabajo, mediante el aprendizaje, siempre está presente el liderazgo y la proactividad de compartir sus ideas con los demás (Begazo \& Fernández, 2016). Así como el de Great Place to Work, en el que se denotan los factores buscados por los millennials, siendo estos una buena remuneración acompañada de la capacidad de desarrollarse con soltura en su puesto y con sus compañeros, sentir un buen ambiente y así no tener que sentirse forzados cuando de ir al trabajo se trate (De la Cruz, 2015). Para ellos es importante trabajar con buenas personas y en un buen ambiente laboral, es decir, saber que cuentan con él y que él cuente con ellos (Ng et al., 2010).

Además, este grupo generacional suelen tener estas características: desafían a sus superiores, esperan ser promovidos rápidamente, quieren ser reconocidos y respetados, son leales a las personas, a sus compañeros de trabajo, a sus jefes pero no tanto a la organización, la familia y los amigos son más importante que el trabajo, trabajar no es lo más importante, son usuarios activos de tecnología, les gusta el cambio, quieren aprender cosas por ellos mismos, valoran el trabajo en equipo, necesitan constantemente feedback para saber si están haciendo bien su trabajo, cómo pueden hacerlo mejor y quieren generar un impacto en la sociedad a través de su trabajo (Zopiatis et al., 2012).

En el personal millennial el desarrollo de un plan de carrera es crítico para lograr que el colaborador permanezca en la empresa, lo cual se convierte en una diferencia competitiva y difícil de replicar (Gutiérrez, 2017). Por ello, los millennials peruanos en el ámbito laboral están en búsqueda del crecimiento constante, que vaya al mismo ritmo con que cambian las cosas en el mundo tecnológico, están dispuestos a transmitir todas sus ideas y aprender todo lo que necesiten para lograr mejores resultados. Buscan un buen ambiente donde trabajar, implicando desde el espacio y si este transmite que la empresa está interesada en el talento que tiene para compartir con ella. 


\section{METODOLOGIA}

Esta investigación tiene un enfoque cualitativo, un estudio de caso de tipo descriptivo explicativo. Es un estudio cualitativo porque se trabajó con una muestra pequeña y se estudió a profundidad. En una investigación cualitativa se observan fenómenos tal como se dan en su contexto natural (The Sage Glossary of the Social and Behavioral Sciences, 2009b); y es apropiada para variables que no pueden o no deben ser manipuladas o resulta complicado hacerlo (Mertens, 2010).

La razón principal por la cual se escogió un estudio de caso fue porque es importante para la obtención de una visión equilibrada de la realidad, ya que nos permite entender mejor la conducta humana, la cual no está regida netamente por reglas o teorías (Flyvbjerg, 2004). Este estudio se sitúa en una empresa peruana del sector inmobiliario, y es un estudio de caso ya que las habilidades blandas no solo varían de acuerdo al puesto laboral, sino que influye también la cultura organizacional y la sociedad (Stasz, 2001). Así como, la mejor comprensión que brinda acerca del entorno estudiado y su complejidad, permitiendo la generación de conocimientos más amplios abriendo puertas a estudios futuros como beneficios del estudio de caso (Cepeda, 2006).

La investigación es descriptiva porque el estudio de las variables se realiza a partir de la recolección de datos sobre una situación específica, en este caso, los millennials que se encuentran laborando en el área de ventas de la empresa Paz Centenario; y a la vez, es una investigación explicativa porque se estudia a profundidad, no solo relatamos un caso en particular, sino que buscamos el por qué impactaría las habilidades blandas en la retención del talento millennial (Abreu, 2012).

Así mismo, respecto a la recolección de datos, esta se realizó a través de entrevistas a once trabajadores millennial, dos supervisores y al jefe del área de ventas; una muestra no probabilística, en este caso, de una empresa peruana del sector inmobiliario en el año 2018. La investigación se elaboró en dos etapas, ya que la retención depende del trabajador y de la empresa. Primero se entrevistaron a dos supervisores y al jefe del área para conocer la opinión al momento de decidir la retención o prescindir de un colaborador. Luego, fueron once trabajadores millennial que forman parte del área de ventas de la empresa, se consideró la edad y tiempo que se encontraban trabajando.

Se consideró relevante conocer la edad que tiene cada trabajador para que vaya de acuerdo con la muestra y pertenezcan a la generación millennial, al mismo tiempo, la antigüedad que llevan trabajando en la empresa, ya que refleja un indicador al tomar en cuenta la retención del talento y la opinión que tienen sobre la empresa. En la siguiente tabla se puede observar los datos por cada colaborador entrevistado.

En Apéndice tabla 1, se puede ver la antigüedad de los millennial en la empresa difieren bastante uno de otro, desde los cuatro meses hasta los nueve años, incluyendo el conocimiento y percepción de la empresa en las variables de estudio. Pero, todos se encuentran en la generación millennial, cumpliendo el rango de edad entre los 23 y 38 años.

El instrumento de recolección de datos (entrevistas), fue semiestructurada, lo cual brindó libertad a los participantes al momento de contestar las preguntas; y también, a no desviarse del tema en cuestión, tanto para los millennial como para los jefes. Las preguntas para ambas muestras fueron elaboradas con siguientes palabras clave: características de los millennials, ambiente laboral, atractividad de la empresa y desarrollo profesional. Estas permitieron agrupar las respuestas tanto de los supervisores como de los millennial de manera homogénea, facilitó la triangulación de datos con la teoría. Entiéndase la triangulación como el procedimiento a través del cual se cruzan más de dos fuentes de datos, en este caso las respuestas de las dos muestras y la teoría, con el fin de validar los resultados encontrados.

Para el análisis de datos cualitativos se siguieron los siguientes pasos: obtener la información; capturar, transcribir y ordenar la información; codificar la información; y finalmente, integrar la información (Fernández, 2006). Las dimensiones asignadas de acuerdo a la 
teoría son: habilidades, actitudes y valores junto con la atractividad de la empresa para la variable de habilidades blandas, y para la variable de retención del talento humano son ambiente laboral y desarrollo profesional. Luego de organizar la información según los pasos antes mencionados, se analizó la relación que existe entre las dimensiones de las variables en cada categoría de los resultados.

\section{CONCERTACIÓN DE PERSPECTIVAS: SUPER VISORES Y MILLENNIALS}

Antes de concertar los resultados de las dos etapas de la investigación, como mencionamos antes, la muestra son trabajadores del área de ventas de una empresa peruana del sector inmobiliario. Esta empresa, tiene diez años en el mercado peruano, se creó a través de un joint venture entre una empresa chilena y una empresa peruana. El giro del negocio es en el sector construcción en especial en condominios, actualmente cuenta con cuatro "proyectos" o condominios, uno en elaboración y otro en lanzamiento. Esta es una empresa mediana, tiene alrededor de ochenta trabajadores, pertenecientes a diferentes grupos generacionales como son la Generación X y la Generación Y o millennials.

A continuación, se presenta la concertación de los resultados de las dos etapas de la investigación mencionadas previamente; de esta forma, las respuestas tanto de la muestra de trabajadores millennials como de jefes de línea de estos trabajadores han sido trianguladas.

\section{Habilidades blandas clave en la retención del talento millennial}

Se observa que entre los jefes, las habilidades resaltantes se pueden relacionar a las cinco habilidades principales mencionadas en la teoría. Se agruparon las habilidades más buscadas, tales como la orientación al conocimiento, el liderazgo, la habilidad de comunicar, los valores éticos y la capacidad de trabajar en equipo. De entre las habilidades que se encuentran en el primer grupo, los jefes mencionaron la capacidad y disposición a aprender, así como la flexibilidad y la proactividad que muestran ante la necesidad de solucionar problemas. Respecto a los grupos de liderazgo y valores éticos, se mencionó la necesidad de que estos colaboradores tengan compromiso con la empresa, de forma que aspiren a el crecimiento conjunto de esta con los colaboradores. Las habilidades de comunicación resaltaron por la necesidad de estas para relacionarse correctamente con los demás, así como que estas les permiten ser más que "máquinas que solo absorben de conocimiento" destinadas a ser "islas llenas de este" sin transmitir sus opiniones ni aportar con la generación de nuevas ideas. Esto influye en la capacidad de trabajar en equipo, lo cual es de gran importancia en esta área, pues se traba en equipos para las diferentes zonas en las que se tiene proyectos. Dependiendo de cómo se dé este trabajo en equipo, se logrará una mejora o de lo contrario un empobrecimiento del ambiente laboral.

Los millennials, al momento de decidir si trabajar en una empresa o no, toman en cuenta factores como el sueldo, el ambiente laboral, la oportunidad de crecimiento y la estabilidad. Sin embargo, a pesar de que el sueldo sea un factor de gran importancia - como lo es para muchos colaboradores de diferentes grupos generacionales - , se puede decir que este no es el más importante. De acuerdo con la cantidad de veces y el orden en que fueron mencionados, el factor de mayor importancia resultó ser el ambiente laboral, seguido de la estabilidad que la empresa le pueda brindar. Los colaboradores hicieron remarque en el primero debido a que, al pasar tanto tiempo en el trabajo, necesitan que este no impacte negativamente en su tranquilidad emocional. Esto va muy relacionado con la estabilidad que se les proporciona; como con la posibilidad de hacer línea de carrera en la empresa. A pesar de la muy extendida creencia que indica que los millennials no buscan asentarse en un puesto, los millennials peruanos en una empresa inmobiliaria reflejan que no es tanto el deseo de moverse constantemente, sino el de encontrar una empresa en la cual el ambiente les permita llevar una vida laboral tranquila y puedan desarrollarse profesionalmente.

Al comparar las respuestas de las dos partes involucradas en la retención, se puede determinar 
que el buen ambiente laboral es uno de los factores de mayor importancia para los millennials, para lo cual se requiere estos cuenten con habilidades de comunicación como la capacidad de relacionarse correctamente con los demás. Esto sumado con actitudes como el compromiso o la afinidad por los retos, logran que su desarrollo profesional sea impulsado, y con esto una mejor oportunidad en hacer una línea de carrera larga.

\section{Elementos buscados en el ambiente laboral por los millennial}

Para los jefes, los elementos buscados por los millennials son la flexibilidad que puedan tener respecto a sus horarios, así como la posibilidad de ser creativos y aportar de esta forma a la empresa. Algunas de las formas en que aportan a la empresa, según los jefes, son debido a habilidades como la facilidad para trabajar en equipo y comunicarse con otros, junto con la empatía y asertividad que demuestran al realizar sus tareas.

Los trabajadores millennials, indican que, entre los elementos vitales para el desarrollo de sus tareas, necesitan el apoyo de sus miembros de equipo, así como de otras áreas junto con las herramientas necesarias. Por consiguiente, consideran que los miembros que formen parte de su equipo, así como sus líderes, deberán saber mantener la compostura, estar abiertos al dialogo y mostrar confianza y empatía con todos.

Cotejando ambos grupos de respuestas, se puede deducir que el ambiente laboral y el correcto trabajo en equipo es clave para el desempeño laboral de los millennials, haciendo que este sea el elemento más buscado en el ambiente laboral por los colaboradores de esta generación.

\section{Influencia de las habilidades blandas en el ambiente laboral}

Los jefes ven al desarrollo de las habilidades blandas como una acción que ayuda al desarrollo diferenciado y trascendente de la empresa, o en otras palabras el nivel de atractividad que esta tiene. Por lo cual cuentan con un esquema económico y de desarrollo profesional, con el cual logran tener, hasta cierto punto, el panorama respecto a cuan desarrolladas se encuentran estas habilidades. También consideran de gran importancia estas habilidades debido a que sirven en la resolución de inconvenientes, así como al enfrentar la jornada laboral de la mejor manera; teniendo en cuenta que uno pasa gran parte del día en su lugar de trabajo.

Los colaboradores millennials mencionan que, al ser partes del área comercial, habilidades como el trato interpersonal es de gran importancia. Indican que, si bien tener los conocimientos es de gran importancia, si no pueden conectarse con los clientes o entablar diálogos, no se puede concretar la venta. Más aún, la experiencia de la venta no termina al salir de la sala de venta, de acuerdo como fue el trato del vendedor, la "reseña" que podrían dar a sus conocidos que se encuentran en busca de una vivienda puede ser negativa o positiva. Para lograr que la experiencia sea la mejor posible, es necesario que los colaboradores cuenten con habilidades como las comunicativas y la empatía, junto con el respeto que se les debe tener a los otros, se logra que el cliente tenga confianza y se puedan conseguir los mejores resultados posibles, aunque no se llegue a concretar la venta.

Estas respuestas nos permiten determinar que la necesidad de las habilidades blandas se da tanto en el ámbito interno de la empresa como el externo. Debido a que esta área tiene constante trato con los clientes, estos dos ámbitos son parte del ambiente laboral de los colaboradores, por lo cual la falta de las habilidades blandas podría afectar negativamente no solo las interacciones con los demás colaboradores, sino también con sus clientes, afectando directamente su nivel de ventas, con lo cual el desempeño de toda la compañía se vería comprometido.

\section{Influencia del desarrollo de habilidades blandas en el desarrollo profesional}

Los jefes de esta área consideran que las habilidades clave con las que todo profesional debe contar para un correcto desarrollo como profesional son las interpersonales, así como las de comunicación. Estos dos tipos de habilidades son más detalladas por los líderes: la empatía para 
tratar tanto con colaboradores como clientes, la capacidad de escucha activa, la comunicación eficaz, comunicación no verbal. Así mismo, consideran que estas deben ser acompañadas de actitudes y valores como la honestidad y la proactividad. Los jefes sostienen que, de contar con estas, el trabajo en equipo se facilita en gran medida.

Los colaboradores millennial consideran que las habilidades necesarias para el desarrollo profesional son las misma que permiten que uno se pueda desempeñar correctamente en el trabajo, y relacionarse con sus compañeros. Por lo cual, de no desarrollar habilidades como asertividad, habilidades de comunicación, inteligencia emocional y habilidades interpersonales, una persona no podrá desarrollarse correctamente en el ámbito profesional. Así mismo, cara al desempeño en las ventas, consideran que, de no potenciarse la capacidad de negociación, los resultados se verán afectados negativamente.

Ambos grupos de entrevistados remarcaron la necesidad de las habilidades interpersonales las de comunicación como las más críticas. Esto se debe a que uno no trabaja solo, sino en equipos y siempre tendrá compañeros, junto con los cuales deberá desempeñarse día a día, y con quienes pasará a mayor parte de esos días. De no saber comunicarse o tratar con los demás, se causan malentendidos que afectan al ambiente laboral, fallas en la comunicación que causan una baja en la productividad, así como malas coordinaciones afectando el trabajo en equipo.

\section{Factores clave en la retención del talento millennial}

A los ojos de los jefes, el clima laboral no es un factor de gran importancia, sino el decisivo, en la retención de los colaboradores millennial. Así mismo, se mencionó la flexibilidad de horarios que se les otorga, de forma que tienen tiempo para sus actividades del ámbito personal. Otro factor que es de relativa importancia es la oportunidad de desarrollo, no solo de línea de carrera, sino de desarrollo profesional de cada uno.

Los millennials, al preguntarles si se encontraban cómodos en el ambiente de trabajo, la respuesta unánime fue sí. Ellos alegan esta comodidad a la comunicación, el compañerismo y la pasión que sus supervisores les han transmitido. Así mismo, dejaron claro su deseo por hacer línea de carrera, y de esta forma crecer junto con la empresa.

Se puede observar, que, a lo largo de las preguntas realizadas, el factor de mayor incidencia es el ambiente laboral; seguido por la oportunidad de crecer profesionalmente.

\section{CONCLUSIONES}

En el caso estudiado indica que los colaboradores millennial deciden continuar trabajando en la empresa si consideran que el ambiente laboral es favorable y tienen oportunidad de realizar línea de carrera. Son estos mismos factores los que resultan clave para una efectiva retención de talento millennial según un estudio realizado a jóvenes peruanos pertenecientes a este cohorte generacional (Begazo \& Fernández, 2015).

Los jefes del área de ventas de la empresa estudiada son conscientes que la generación $\mathrm{Y}$ será la que dirija el mundo laboral en unos años y están dispuestos a trabajar con ellos. Es trabajando juntos que se crea la oportunidad de compartir conocimientos e ideas entre miembros de distintas generaciones, logrando de esta forma mejores resultados.

Los elementos buscados en el ambiente laboral por los trabajadores millennial son el trabajo en equipo y compartir los mismos objetivos, de forma que se logren las metas del área, siempre manteniendo la confianza y el respeto mutuo. Así mismo, son habilidades blandas como la orientación al conocimiento, habilidad para comunicar, lograr los objetivos con ética y capacidad de trabajar en equipo las que facilitan el relacionarse con diferentes tipos de personas. Siendo así necesario que estas habilidades estén presentes en todos los colaboradores para mantener un buen ambiente laboral (Puga \& Martínez, 2008).

La influencia de las habilidades blandas en el desarrollo profesional, en el caso estudiado, se 
nota a nivel de la técnica de ventas. Esta ha sido perfeccionada a través de la empatía y la comunicación, con lo cual se consigue no solo cumplir con el objetivo, si no que el cliente deposite su confianza en ellos. Para el logro de los objetivos es necesario trabajar en equipo, la honestidad y la integridad, así como estar dispuesto a escuchar opiniones de los otros. Estas habilidades son potenciadas con el fin de conseguir un buen ambiente laboral. De igual manera, se entiende que la búsqueda de los millennials peruanos por el crecimiento constante, no se limita a ascensos, sino que comprender también el aprender, desarrollar diferentes habilidades y poder transmitir sus ideas a los demás (Singer, Guzmán \& Donoso,2009).

Es así que las habilidades que resaltan entre las principales buscadas en el personal, son las de comunicación y la capacidad de trabajar en equipo, seguidos por la orientación al conocimiento y valores éticos (Puga \& Martínez, 2008). Asimismo, pueden ser observadas como claves en la retención de talento millennial peruano, por el caso estudiado, debido a su imprescindibilidad de un buen ambiente laboral y desarrollo profesional. De esta forma, habilidades que faciliten el relacionamiento con otros y el trabajo en equipo así como la flexibilidad, proactividad y compromiso que muestren, son las más buscadas por y en el colaborador millennial.

En consecuencia, a los puntos antes expuestos, quien cuente con estas habilidades blandas tiene mayor probabilidad de permanecer en la empresa. Esto se debe a que las habilidades del "saber ser" son necesarias en el trato con otras personas (Russell \& Carol, 2000). Es coherente que sean las habilidades comunicativas y de relación interpersonal las esenciales para un buen ambiente laboral; el cual es muy cuidado debido a que de faltar o no ser óptimo, la salud del trabajador se vería afectada, causando que este busque un nuevo lugar de trabajo. Esto va de acuerdo con lo que Guerrero et al. (2006) plantearon sobre la necesidad que el ambiente laboral vele por el bienestar mental y físico de sus colaboradores.

Finalmente, debido a que las habilidades blandas influyen en la interacción interpersonal, generando la calidad el ambiente laboral, la cual fue demostrada como el factor de mayor importancia en la retención de talento millennial; se puede concluir que estas son de gran importancia para la retención de talento millennial peruano.

De acuerdo con lo concluido, se recomienda para próximas investigaciones que el grupo seleccionado como muestra sea de mayor tamaño, debido a que permitirá la obtención de una mayor cantidad de puntos de vista y de información. De buscar como resultado una generalización o la comprobación de una hipótesis, se recomienda realizar el estudio en dos o más empresas cuyas características sean similares. Las diferencias entre esta podrían brindar mayor data comparativa; sin embargo, de ser estas diferencias muy marcadas, es probable que los resultados obtenidos no lleguen a un consenso. Por último, se recomienda optar por un estudio longitudinal para obtener de forma más completa los efectos que tenga a largo plazo la aplicación de las habilidades blandas como estrategia para retención del talento millennial.

\section{REFERENCIAS}

Acosta, C. (2017). Motivaciones laborales de Millennials que trabajan en diversas empresas de la ciudad de Bogotá, Colombia. Universidad de La Sabana, Chía, Colombia.

Ahmed, P.K., Loh, A. y Zairi, M. (1999). Cultures for continuous improvement and learning. Total Quality Management, 10(4-5), 426- 434.

Allen, D., Bryant, P., \& Vardaman, J. (2010). Retaining Talent: Replacing Misconceptions With Evidence-Based Strategies. Academy of Management Perspectives, 24(2), 48-64. Retrieved from http://www.jstor.org/stable/25682398

Begazo Villanueva, J., \& Fernandez Baca, W. (2016). Los Millennials Peruanos: Características y Proyecciones de vida. Gestión en el Tercer Milenio, 18(36), 9-15. ISSN: 17282969 Recuperado de http://revistasinvestigacion.unmsm.edu.pe/index. php/administrativas/article/view/11699/104 89 
Bergerman, E. \& Tantaleán, S. (2016). Propuesta de retención del talento millennials en la entidad financiera Banco de Crédito del Perú en el departamento de Lambayeque - 2016. Universidad Católica Santo Toribio de Mogrovejo, Chiclayo, Perú.

Cepeda, C. (2006). La calidad en los métodos de investigación cualitativa: Principios de aplicación práctica para estudio de casos. Cuadernos de Economía y Dirección de la Empresa. (ACEDE), (29), 57-82. Recuperado de https://dialnet.unirioja.es/descarga/articulo/2385 980.pdf

De la Cruz, C. (2015). Millennials en el Perú: Una generación con grandes oportunidades laborales. Bolsa de trabajo PUCP, de la Pontificia Universidad Católica del Perú. Recuperado de https://btpucp.pucp.edu.pe/archivoreportajes/millennials-en-el-peru-unageneracion-con-gr andes-oportunidadeslaborales/

EMPRESAS. $43 \%$ de sobrecostos sería por mala selección de personal. (2016, 23 de julio). La República. Recuperado de https://larepublica.pe/economia/788014empresas-43-de-sobrecostos-seria-por-malaselecci on-de-personal

Fernández Núñez, L. (2006). Ficha para investigadores: ¿Cómo analizar datos cualitativos? Butlletí LaRecerca, (7) ISSN: 18861946 Recuperado de

http://www.ub.edu/ice/recerca/pdf/ficha7cast.pdf

Finn, D. \& Donovan A. (2013) PwC's NextGen: A global generational study 2013 Summary and compendium of findings. Recuperado de https://www.pwc.com/gx/en/hrmanagement-services/pdf/pwc-nextgen-study2013.pdf

Flyvbjerg, B (2004). Cinco malentendidos acerca de la investigación mediante los estudios de caso. Revista Española de Investigaciones Sociológicas (REIS), Abril-Junio 2004, pp. 33-62(30). Recuperado de https://www.ingentaconnect.com/content/cis/reis

\section{/2004/00000106/00000001/art00002\#Refs}

Gordon, M.(2016). Determinación de rasgos distintivos dentro del grupo demográfico denominado los millennials en la ciudad de Guayaquil. Universidad de Especialidades Espíritu Santo, Samborondón, Ecuador.

Goyenechea, D. (2016). Las motivaciones y expectativas laborales de la Generación " $Y o$ Millennials". Análisis en el contexto de una empresa de servicios privada argentina. Universidad de San Andrés, Buenos Aires, Argentina.

Guerrero Pupo, Julio C, Cañedo Andalia, Rubén, Rubio Rodríguez, Samara M, Cutiño Rodríguez, Marieta, \& Fernández Díaz, Delio J. (2006). Quality of life and work: Some considerationson the working environment of the office. ACIMED, 14(4) Recuperado de http://scielo.sld.cu/scielo.php?script=sci_arttext \&pid=S1024-94352006000400005\&lng=es \&tlng $=$ en.

Gutiérrez, L. (2017). Beneficios laborales, económicos y sociales y su impacto en la retención del personal millennial del sector bancario - Lima 2017. Universidad San Ignacio de Loyola, Lima, Perú.

Hershatter, A., \& Epstein, M. (2010). Millennials and the World of Work: An Organization and Management Perspective. Journal of Business and Psychology, 25(2), 211-223. Retrieved from http://www.jstor.org/stable/40605780

Huyler, D., Pierre, Y., \& Ding, W. (Junio, 2015). Millennials in the Workplace : Positioning Companies for Future Success. En S. Nielsen (Presidencia), SFERC. Conferencia llevada a cabo en Florida International University Miami, Florida, Estados Unidos.

I, Chiavenato (2009) Gestión del talento Humano. Recuperado de http://www.academia.edu/download/34860731/L ECTURA_CLASE1_Gestion_del_Talento _Humano.doc

Latukha, M. (2011). To stay or leave: Motives 
behind the decisions of graduate programs' trainees' in European and Russian companies. Journal of East European Management Studies,16(2), 140-161. Retrieved from http://www.jstor.org/stable/23281853

Levasseur, R. (2013). People Skills: Developing Soft Skills-A Change Management Perspective.

Interfaces, 43(6), 566-571. Retrieved from http://www.jstor.org/stable/43699350

Lima, J. \& Polanco, F. (2017). Satisfacción laboral en los millennials en comparacion a la generación " $X$ " en Arequipa, 2017. Universidad Católica San Pablo, Arequipa, Perú.

Mertens (2010). Capítulo 7: Concepción o elección del diseño de investigación. En R, Hernández $\left(6^{\circ}\right.$ edición $)$ Metodología de la investigación (pp. 153) México DF: INTERAMERICANA EDITORES.

Meza,Y. (2016). El branding y la fidelización de clientes millennials en el BBVA Banco Continental, Cercado de Lima - 2016. Universidad César Vallejo, Lima, Perú.

Myers, K. K. \& Sadaghiani, K (2010). Millennials in the Workplace: A Communication Perspective on Millennials' Organizational Relationships and Performance. Journal of Business and Psychology, 25(2), 225-238. doi: 10.1007/s10869-010-9172-7

Ng, E., Schweitzer, L., \& Lyons, S. (2010). New Generation, Great Expectations: A Field Study of the Millennial Generation. Journal of Business and Psychology, 25(2), 281-292. Retrieved from http://www.jstor.org/stable/40605786

Pacheco, J. \& Ríos, A. (2017). El millennial peruano como cliente interno y externo del sector retail. Universidad Peruana de Ciencias Aplicadas, Lima, Perú.

Paulone, A. \& Pulice, A. (2012). Motivación y retención de jóvenes profesionales y estudiantes universitarios pertenecientes a la Generación $Y$ en grandes empresas. Universidad Argentina de la Empresa (Fundación UADE), Buenos Aires,
Argentina.

PEARLMAN, L., \& BARRETT, B. (2012). Six New Skills for Today's Frontline Leader.

Perspectives on Work, 16(1/2), 54-57. Retrieved from http://www.jstor.org/stable/41810211 Pérez López, J. A. (1997) (edición póstuma). Liderazgo. Barcelona: Folio.

Pierce, L. (2018, 11 de junio). Los 'millennials', mucho más que simples consumidores. El Comercio - Díal, p.22

PriceWaterhouseCooper S. Civil de R.L. (2014) ler estudio: Tendencias de retención del talento Perú 2014 Un desafío para las organizaciones de hoy. Recuperado https://www.pwc.pe/es/publicaciones/assets/1erestudio-de-retencion-de-talento-peru-2014. pdf

Puga Villarreal, J., \& Martínez Cerna, L. (2008). Competencias directivas en escenarios globales.

Estudios Gerenciales, 24 (109), 87-103.

Salas, R. (2017). La Inteligencia Emocional de los Colaboradores "Millennials" en la Empresa Ventcorp-Perú. Universidad Inca Garcilaso de la Vega, Lima, Perú.

Singer, M., Guzmán, R. \& Donoso, P. (2009) Entrenando Competencias Blandas en Jóvenes.

Escuela de Administración Pontificia Universidad Católica de Chile

Stasz, C. (2001). Assessing Skills for Work: Two Perspectives. Oxford Economic Papers, 53(3), 385-405. Retrieved from http://www.jstor.org/stable/3488625

The Sage Glossary of the social and Behavioral Sciences (2009b) Capítulo 7: Concepción o elección del diseño de investigación. En R, Hernández $\left(6^{\circ}\right.$ edición) Metodología de la investigación (pp. 152) México DF: INTERAMERICANA EDITORES

The Soft Skills Disconnect (2015, 13 de febrero). National Soft Skills Association. Recuperado de https://www.nationalsoftskills.org/the-soft-skills- 
disconnect/

Tito, M. \& Serrano, B. (2016) Desarrollo de soft skills una alternativa a la escasez de talento humano. INNOVA Research Journal, 1(12), 5976. ISSN 2477-9024. Recuperado de https://dialnet.unirioja.es/descarga/articulo/5920 579.pdf

Weber, M., Finley, D., Crawford, A., \& Rivera, D. (2009). An exploratory study identifying soft skill competencies in entry-level managers. Tourism and Hospitality Research, 9(4), 353-361.
Retrieved

from

http://www.jstor.org/stable/23745488

Zopiatis, A., Krambia-Kapardis, M., \& Varnavas, A. (2012). Y-ers, X-ers and Boomers: Investigating the multigenerational (mis)perceptions in the hospitality workplace. Tourism and Hospitality Research, 12(2), 101121. Retrieved from http://www.jstor.org/stable/43498600 


\section{APÉNDICES}

TABLA 1

Características de los millennial del área de ventas de la empresa

\begin{tabular}{ccc}
\hline Colaborador & Edad & $\begin{array}{c}\text { Antigüedad en la empresa } \\
\text { (expresado en meses) }\end{array}$ \\
\hline Colaborador 1 & 25 & 12 \\
Colaborador 2 & 36 & 12 \\
Colaborador 3 & 31 & 6 \\
Colaborador $\mathbf{4}$ & 32 & 24 \\
Colaborador 5 & 32 & 4 \\
Colaborador 6 & 32 & 108 \\
Colaborador 7 & 27 & 18 \\
Colaborador 8 & 27 & 36 \\
Colaborador $\mathbf{9}$ & 31 & 18 \\
Colaborador $\mathbf{1 0}$ & 30 & 8 \\
Colaborador 11 & 34 & 30 \\
\hline
\end{tabular}

